\title{
The Effect of Adding Kami-guibi-tang to Acetylcholinesterase Inhibitor Treatment on the Cognitive Function of Mild Alzheimer's Disease Patients: Study Protocol of a Randomized, Placebo-Controlled, Double-Blind Pilot Trial
}

\author{
Seung-bo Yang' ${ }^{1}$ Ha-ri Kim.3. ${ }^{2.3}$ Hee-yeon Shin², Jeong-hwa Kim², Chang-woo Lee , \\ Geon-ho Jahng ${ }^{4}$, Seong-uk Park ${ }^{2.5}$, Chang-nam Ko $0^{2.5}$, Jung-mi Park ${ }^{2.5}$ \\ 'Dept. of Korean Internal Medicine, College of Korean Medicine, Gachon University \\ ${ }^{2}$ Stroke and Neurological Disorders Center, Kyung Hee University Hospital at Gangdong \\ ${ }^{3}$ Dept. of Clinical Korean Medicine, Graduate School, Kyung Hee University \\ ${ }^{4}$ Dept. of Radiology, Kyung Hee University Hospital at Gangdong, College of Medicine, Kyung Hee University \\ ${ }^{5}$ Dept. of Cardiology and Neurology, College of Korean Medicine, Kyung Hee University
}

\section{The Effect of Adding Kami-guibi-tang to Acetylcholinesterase Inhibitor Treatment on the Cognitive Function of Mild Alzheimer's Disease Patients: Study Protocol of a Randomized, Placebo-Controlled, Double-Blind Pilot Trial}

\author{
Seung-bo Yang1, Ha-ri Kim, ${ }^{2.3}$ Hee-yeon Shin², Jeong-hwa Kim², Chang-woo Lee ${ }^{3}$, \\ Geon-ho Jahng ${ }^{4}$, Seong-uk Park ${ }^{2.5}$, Chang-nam Ko ${ }^{2.5}$, Jung-mi Park ${ }^{2.5}$ \\ 'Dept. of Korean Internal Medicine, College of Korean Medicine. Gachon University \\ ${ }^{2}$ Stroke and Neurological Disorders Center, Kyung Hee University Hospital at Gangdong \\ ${ }^{3}$ Dept. of Clinical Korean Medicine, Graduate School, Kyung Hee University \\ ${ }^{4}$ Dept. of Radiology, Kyung Hee University Hospital at Gangdong, College of Medicine, Kyung Hee University \\ ${ }^{5}$ Dept. of Cardiology and Neurology, College of Korean Medicine, Kyung Hee University
}

\begin{abstract}
Background: Alzheimer's disease (AD) is a chronic neurodegenerative disease that causes disorientation, mood swings, problems with language, and difficulty remembering recent events. Acetylcholinesterase inhibitors (AchEIs) and memantine have been used to slow the course of the disease, but they can neither modify its progression nor prevent disease onset. Previous studies have suggested that Kami-guibi-tang (KGT) could be beneficial for supporting cognitive function in AD patients, but few clinical trials have been published. This pilot study aimed to evaluate the effect of $\mathrm{KGT}$ in improving cognitive function in AD patients.

Methods: The study will be a randomized, placebo-controlled, double-blind, single-center trial conducted using subjects diagnosed with mild AD by neurologists. Study subjects will be randomly assigned to either a treatment or control group. The treatment group will receive KGT granules for 24 weeks, while the control group will receive placebo granules. AchEI administration will be maintained in both groups during the entirety of the study. Subjects will be assessed using the following exams: the Seoul Neuropsychologic Screening Battery (SNSB) for cognitive function; brain magnetic resonance imaging (MRI) for brain metabolite, neurotransmitter, and cerebral blood flow (CBF) measurements; the Korean version of Quality of Life-Alzheimer's Disease (KQol-AD) for quality of life; the Caregiver-Administered Neuropsychiatric Inventory (CGA-NPI) for neurobehavioral symptoms: blood tests for amyloid and tau proteins and general blood parameters; and electrocardiography (ECG) before and after taking the medication. Discussion: Our findings will provide insight into the feasibility of large-scale trials to consolidate evidence for the efficacy of KGT for dementia treatment.

Registration ID in CRIS: KCT0002904 (Clinical Research Information Service of the Republic of Korea).
\end{abstract}

Key words: mild Alzheimer's disease, Kami-guibi-tang, herbal medicine, Seoul neuropsychological screening battery (SNSB), magnetic resonance imaging (MRI)

· 투고일 : 2020.03.30, 심사일: 2020.07.03, 게재확정일: 2020.07.03

- Corresponding author: Jung-mi Park Stroke and Neurological Disorders Center, Kyung Hee University Hospital at Gangdong, 892, Dongnam-ro, Gangdong-gu, Seoul, Republic of Korea TEL: 02-440-6216 FAX: 02-440-7171 E-mail: pajama@khu.ac.kr

\section{Introduction}

Alzheimer's disease $(\mathrm{AD})$ is a chronic neurodegenerative disease that causes difficulty in remembering recent 
events, problems with language, disorientation, and mood swings ${ }^{1}$. AD typically progresses slowly and in three general stages; mild, moderate, and severe ${ }^{2}$. Neuropsychiatric symptoms are common even in the early stages of $\mathrm{AD}$, and are associated with a reduction in quality of life. The economic impact of dementia is estimated at $\$ 600$ billion per year globally, which is greater than the economic impact of other common chronic diseases ${ }^{4}$. Currently, there are two classes of drugs approved for the treatment of $\mathrm{AD}$ : acetylcholinesterase inhibitors (AChEIs) are effective for improving cognition in mild to moderate $\mathrm{AD}$, and memantine is used for the treatment of moderate to severe $\mathrm{AD}^{5}$.

Kami-guibi-tang (KGT) is an herbal medicine commonly used in Korean medicine, Kampo medicine (Kamikihi-to), and traditional Chinese medicine. KGT is a modified version of Guibi-tang. Guibi-tang has been shown to exhibit myriad effects, including anti-stress $^{6}$, anti-oxidant ${ }^{7}$, hemostasis ${ }^{8}$, anti-osteoporosis ${ }^{9}$, gastroprotection $^{10}$, reproductive ability ${ }^{11}$, brain cell activity $^{12}$, and radioprotective effects ${ }^{13}$, in biological studies. Guibi-tang treatment has also been shown to lead to improvements in memory and orientation, insomnia, varicose veins, and schizophrenia in clinical studies ${ }^{14}$.

Several studies have explored the effects of KGT on cognitive impairment. KGT and donepezil combination therapy may prolong the effect of donepezil in patients with $\mathrm{AD}^{15}$ and KGT may improves cognitive function and activities of daily living in patients with $\mathrm{AD}$ and vascular dementia ${ }^{16}$. The results of animal studies suggest that KGT improves impairment of spatial memory ${ }^{17}$, rescues axonal and synaptic degeneration associated with memory impairment ${ }^{18}$, and improves cognitive impairment by reducing neuronal apoptosis and $\mathrm{A}$ $\beta$ accumulation in the hippocampus ${ }^{19}$. In addition, the neuroprotective effects and memory-enhancing effects of extracts of radix ginseng ${ }^{20}$, radix polygalae ${ }^{21}$, radix angelicae $e^{22}$, and radix glycyrrhizae $e^{23}$, which are herbs containing KGT, have been studied extensively.

AChEIs slow the course of AD, but cannot modify its progression or prevent onset. Currently, there are no licensed disease-modifying agents for $\mathrm{AD}$ nor any that are recommended for clinical use $^{5}$. Although previous studies have suggested that KGT may be beneficial for improving cognitive function in $\mathrm{AD}$ subjects, few randomized controlled trials have been conducted. This manuscript describes the protocol of a pilot randomized controlled trial designed to evaluate the effect of adding KGT to AchEI treatment on the cognitive function of $A D$ patients, and its influence on neuroimaging and neurochemical biomarkers.

\section{Methods}

\section{Study design and setting}

This study was designed as a randomized, placebocontrolled, parallel-group, double-blind, single-center trial to be conducted at Kyung Hee University Hospital at Gangdong, Seoul, Korea, from January 1st, 2018 to November 30th, 2019. The flow chart of the study design is shown in Fig. 1. The schedules for enrollment, interventions, and assessments are shown in Fig. 2. This study follows the recommendations of the Standard Protocol Items of Recommendations for Interventional Trials (SPIRIT) Checklist. 
The Effect of Adding Kami-guibi-tang to Acetylcholinesterase Inhibitor Treatment on the Cognitive Function of Mild Alzheimer's Disease Patients: Study Protocol of a Randomized, Placebo-Controlled, Double-Blind Pilot Trial

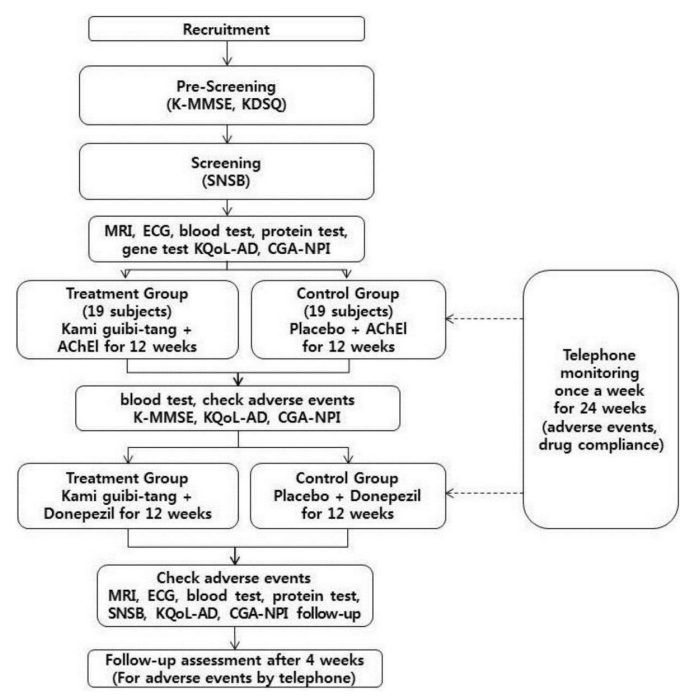

Fig. 1. Flow chart of the study design.

K-MMSE : Korean version of the mini-mental state examination, KDSQ : Korean dementia screening questionnaire, SNSB : Seoul neuropsychological screening battery, MRI : magnetic resonance imaging, ECG : electrocardiogram, KQoL-AD : Korean version of the quality of life-Alzheimer's disease, CGA-NPI : caregiver-administered neuropsychiatric inventory, AChEI : acetylcholinesterase inhibitor

\begin{tabular}{|c|c|c|c|c|c|c|}
\hline \multirow[b]{3}{*}{ TIMEPOINT } & \multicolumn{6}{|c|}{ STUDY PERIOD } \\
\hline & \multirow{2}{*}{\begin{tabular}{|c|c|}
$\begin{array}{c}\text { Pre- } \\
\text { screening }\end{array}$ \\
$-2 \sim \theta$ \\
weeks
\end{tabular}} & \multirow{2}{*}{$\begin{array}{c}\text { Screening } \\
0\end{array}$} & \multicolumn{3}{|c|}{$\begin{array}{c}\text { Treatment } \\
\text { period }\end{array}$} & \multirow{2}{*}{\begin{tabular}{|c|}
$\begin{array}{c}\text { Follow-up } \\
\text { period }\end{array}$ \\
$28^{\text {th }}$ week \\
\end{tabular}} \\
\hline & & & $I^{\prime \prime} d a y$ & $\begin{array}{l}12^{\text {tin }} \\
\text { week }\end{array}$ & $2 f^{2 / 2}$ & \\
\hline \multicolumn{7}{|l|}{ ENROLLMENT: } \\
\hline \multirow{3}{*}{$\begin{array}{r}\text { Eligibility screen } \\
\text { K-MMSE } \\
\text { KDSQ }\end{array}$} & $\mathrm{x}$ & $\mathrm{x}$ & $\mathrm{x}$ & & & \\
\hline & $\mathrm{x}$ & & & $\mathrm{x}$ & & \\
\hline & $\mathrm{x}$ & & & & & \\
\hline \multirow{2}{*}{$\begin{array}{r}\text { Informed consent } \\
\text { SNSB }\end{array}$} & & $\mathrm{x}$ & & & & \\
\hline & & $\mathrm{x}$ & & & $\mathrm{x}$ & \\
\hline NINCDS-ADRDA & & $\mathrm{x}$ & & & & \\
\hline \multirow{2}{*}{$\begin{array}{l}\text { KQoL-AD } \\
\text { CGA-NPI }\end{array}$} & & & $\mathrm{x}$ & $\mathrm{x}$ & $\mathrm{x}$ & \\
\hline & & & $\mathrm{x}$ & $\mathrm{x}$ & $\mathrm{x}$ & \\
\hline Allocation & & & $\mathrm{x}$ & & & \\
\hline \multicolumn{7}{|l|}{ INTERVENTIONS: } \\
\hline \multicolumn{7}{|l|}{ Kami guibi-tang } \\
\hline \multicolumn{7}{|l|}{ Placebo } \\
\hline $\begin{array}{l}\text { Acetylcholinesterase } \\
\text { inhibitor }\end{array}$ & & & & & & \\
\hline
\end{tabular}

\begin{tabular}{|c|c|c|c|c|}
\hline \multicolumn{5}{|l|}{ ASSESSMENTS: } \\
\hline MRI & $\mathrm{x}$ & & $\mathrm{x}$ & \\
\hline$E C G$ & $\mathrm{x}$ & & $\mathrm{x}$ & \\
\hline Blood test (safety) & $\mathrm{x}$ & $\mathrm{x}$ & $\mathrm{x}$ & \\
\hline $\begin{array}{c}\text { Blood test } \\
\text { (proteins, } \\
\text { cholesterol) }\end{array}$ & $\mathrm{x}$ & & $\mathrm{x}$ & \\
\hline Gene test & $\mathrm{x}$ & & & \\
\hline $\begin{array}{r}\text { Monitoring of } \\
\text { adverse events (by } \\
\text { telephone) }\end{array}$ & & & & $\mathrm{x}$ \\
\hline
\end{tabular}

Fig. 2. The schedule of enrollment, interventions, and assessments (SPIRIT 2013 statement).

K-MMSE : Korean version of the mini-mental state examination, KDSQ : Korean dementia screening questionnaire, SNSB : Seoul neuropsychological screening battery, NINCDS-ADRDA : national institute of neurological and communicative disorders and stroke and Alzheimer's disease and related disorders association, KQoL-AD : Korean version of the quality of life-Alzheimer's disease, CGA-NPI : caregiver-administered neuropsychiatric inventory, MRI : magnetic resonance imaging, ECG : electrocardiogram

\section{Participants}

1) Inclusion criteria

Subjects who meet the following criteria will be eligible to participate:

(1) Subjects aged 55-90 years, with complaints of impaired memory.

(2) Subjects diagnosed with mild $\mathrm{AD}$ by a neurologist, following the SNSB (Seoul Neuropsychologic Screening Battery), and NINCDS-ADRDA (National Institute of Neurological and Communicative Disorders and Stroke and Alzheimer's Disease and Related Disorders Association) criteria.

- CDR (Clinical Dementia Rating) $=0.5-1$

- Since there are no absolute values of the K-MMSE (Korean version of the Mini-Mental State Examination), GDS (Global Deterioration Scale), or CDR validated for the diagnosis of mild $A D$, different criteria are applied in different clinical studies. This study includes subjects who are regarded as having mild AD, as assessed with 
the CDR, among patients diagnosed with $\mathrm{AD}$ by neurologists using NINCDS-ADRDA.

(3) Subjects who have received cognitive-related medication, including AChEIs such as donepezil, rivastigmine, and galantamine, and remained stable condition without any adverse events. Cognitiverelated medications include cerebral blood flow improvement agents and other brain nutrients that affect brain cognition, such as gliatilin, gliatamin, ginexin, and tanamine.

(4) Subjects who have been stable for the past two weeks or more without any changes in the medications used for treating their underlying disease, and who are expected to remain stable during this clinical trial.

(5) Subjects who have no communication problems.

(6) Subjects who are not disqualified for reasons related to magnetic resonance imaging (MRI).

2) Exclusion criteria

Subjects who meet any of the following criteria will be ineligible to participate:

(1) Subjects with brain disorders that cause neurological symptoms other than cognitive dysfunction.

(2) Subjects with degenerative brain diseases, such as Parkinson's disease, Huntington's chorea, Down syndrome, and Creutzfeldt-Jakob disease upon history and neurological examinations.

(3) Subjects with cerebral trauma due to hypoxia, such as carbon monoxide poisoning, vitamin deficiency, infectious diseases such as neurosyphilis or encephalitis, brain tumors, endocrine metabolic diseases, or mental retardation.

(4) Subjects with evidence of clinically evident cerebrovascular disease or suspicion of territorial infarct of cerebral blood vessels due to multiple strokes on MRI.

(5) Subjects with a history of convulsive disease (except for febrile convulsions in childhood).

(6) Subjects with a history of depression or who are currently suffering from depression.

(7) Subjects with mental illnesses or behavioral problems requiring psychotropic medications.

(8) Subjects with physical disabilities that are life threatening and require immediate treatment.

(9) Subjects with uncontrolled hypertension.

(10) Subjects with heart or kidney disease.

(11) Subjects with edema.

(12) Subjects with gastrointestinal symptoms (e.g. anorexia, stomach discomfort, nausea, abdominal pain, diarrhea).

(13) Subjects who are taking medications that may cause hypokalemia or myopathy.

(14) Subjects with hypersensitivity to components of clinical trial medicines.

(15) Subjects who might be pregnant.

(16) Subjects with clinically significant abnormalities in their blood chemistry [SGPT (serum glutamic pyruvic transaminase)/SGOT (serum glutamic oxaloacetic transaminase)] more than two times the normal upper limit or serum creatinine 10\% of the normal upper limit).

(17) Subjects who participated in other clinical trials within the four weeks prior to enrollment in the present study.

(18) Illiterate subjects.

(19) Subjects who were taking NMDA receptor antagonists, such as memantine.

(20) Subjects who were deemed inadequate by the tester.

3) Drop-out criteria

(1) Occurrence of any severe adverse effects.

(2) Subject's voluntary withdrawal from the trial.

(3) Subjects not following the protocol (i.e., 
The Effect of Adding Kami-guibi-tang to Acetylcholinesterase Inhibitor Treatment on the Cognitive Function of Mild Alzheimer's Disease Patients: Study Protocol of a Randomized, Placebo-Controlled, Double-Blind Pilot Trial

poor drug compliance, below $80 \%$ ).

(4) Use of additional medication to improve cognitive function during the study period.

(5) Decision made at the discretion of the principal investigator.

\section{Recruitment and enrollment}

A total of at least 38 subjects will be recruited through advertisements and referrals. Candidates with complaints of impaired memory who are aged 55-90 years will be screened using the inclusion /exclusion criteria, K-MMSE, and Korean Dementia Screening Questionnaire (KDSQ). Potentially eligible subjects will undergo the SNSB, and those who are diagnosed with mild $\mathrm{AD}$ by a neurologist will be included in the trial. The purpose, procedures, and potential risks and benefits of the study will be explained to the subjects in detail. Written informed consent and additional informed consent for providing biological specimens will be obtained prior to enrollment.

\section{Randomization, allocation, blinding}

A random, computer-generated, allocation list will be generated using SPSS version 18 by an assigned researcher not involved in the cognitive assessments. The subjects will be randomly allocated to either the treatment or control group in a 1:1 ratio using a block randomization method with a block size of four. Subjects, the assessor, the clinical trial pharmacist, and the researcher will be blinded to allocations throughout the course of the study. If serious adverse events (SAEs) occur, we will unblind the affected cases.

\section{Intervention}

After randomization, the treatment group will receive KGT granules ( $\mathrm{g} / 1$ pack), and the control group will receive placebo granules $(3 \mathrm{~g} / 1$ pack). AChEI administration will be maintained at a constant dose in both groups during the study. Doses of donepezil will be administered on an individual basis.

KGT is composed of 14 drugs: radix astragali $(1.0 \mathrm{~g})$, radix ginseng $(1.0 \mathrm{~g})$, rhizoma atractylodis $(1.0 \mathrm{~g})$, Poria cocos $(1.0 \mathrm{~g})$, zizyphi fructus $(0.67 \mathrm{~g})$, rhizoma zingiberis $(0.33 \mathrm{~g})$, radix saussureae $(0.33 \mathrm{~g})$, radix glycyrrhizae $(0.33 \mathrm{~g})$, zizyphi spinosi semen $(1.0 \mathrm{~g})$, longan arillus $(1.0 \mathrm{~g})$, radix angelicae $(0.67 \mathrm{~g})$, radix polygalae $(0.67 \mathrm{~g})$, radix bupleuri $(1.0 \mathrm{~g})$, moutan radicis cortex $(0.67 \mathrm{~g})$, and gardeniae fructus $(0.67 \mathrm{~g})$. The treatment granules were manufactured by Kyoung Bang Pharmaceutical Co., Ltd (Incheon, Korea), which has been certified to follow the Good Manufacturing Practice guidelines.

The placebo granules will be produced by the same manufacturer, using the standard method of placebo manufacturing according to Korean Good Manufacturing Practice guidelines. The placebo granules are composed of 7 ingredient: corn starch (KP, the Korean Pharmacopoeia) (2,000 mg), lactose (KP) (858.6625 mg), hydroxypropyl cellulose (KP) (120 mg), caramel coloring $(19.35 \mathrm{mg})$, yellow No. 4 (KPTaCS, Korean Phamaceutical Tar Color Specification)(1.2 $\mathrm{mg}$ ), red No. 40 (KPTaCS) $(0.225 \mathrm{mg})$, ssangwha scent $(0.5625 \mathrm{mg})$. The placebo will be similar to the KGT granules in appearance, taste, and smell.

The treatment and placebo granules will be distributed to subjects by an independent clinical pharmacist. The subjects will be instructed to dissolve the granules in hot water, stir well, and drink the solution three times per day, 30 minutes after meals, for 24 consecutive weeks. A reminder phone call will be made every week in order to 
promote adherence to taking the study medication.

Medication for underlying diseases, such as hypertension and diabetes mellitus, will be permitted during the study period. However, any medication that could affect cognitive function will be prohibited. Subjects will be asked to report all medications taken during the study at each visit. The names, duration, and dosage of all drugs will be recorded on the case report form (CRF) of each subject.

The subjects will be required to return any unused trial drugs at the final follow-up visit. Drug compliance will be evaluated by quantifying the returned drugs. Subjects with poorer than $80 \%$ compliance will be excluded from the study.

\section{Assessments}

1) K-MMSE, SNSB, KQoL-AD, and CGA-NPI

The K-MMSE will be administered at the screening visit and after 12 weeks of treatment with either KGT or the placebo to screen for mild $\mathrm{AD}$ and to assess the cognitive status of the subjects. It is a simple, commonly used screening test for longitudinal assessment of general cognition.

The full version of the SNSB-II will be administered at baseline and after 24 weeks of KGT or placebo treatment to evaluate the effect of KGT on cognitive function. The estimated completion time of the whole battery is 1.5-2 hours.

The KQDL-AD and CGA-NPI will be administered at baseline, and after 12 and 24 weeks of treatment with either KGT or placebo. The KQoL-AD is used to assess quality of life in elderly subjects with dementia, and the CGA-NPI is used to assess neuropsychiatric symptoms in dementia patients.

2) MRI

All subjects will undergo brain MRI at baseline and after 24 weeks of KGT or placebo treatment to measure changes in metabolites and neurotransmitter levels, cerebral blood flow, and tissue volume, and to evaluate brain abnormalities.

Proton magnetic resonance spectroscopy (1H-MRS) will be used to measure brain metabolites and neurotransmitter levels. Single-voxel Point-RESolved Spectroscopy (PRESS) MRS will be performed in the precuneus and posterior cingulate areas in the brain with a voxel size of $30 \mathrm{~mm} \times 30 \mathrm{~mm} \times 30$ $\mathrm{mm}$ to detect $N$-acetylaspartate (NAA) and glutamate-glutamine complex ( Glx, with both Glu and Gln) levels. NAA and Glx will be quantified using LCModel software. MEshcher-Garwood (MEGA) PRESS MRS will be performed to detect gammaaminobutyric acid (GABA) in the same area. The amount of GABA will be quantified using GANNET software.

Pseudo-continuous arterial spin labeling (pCASL) MRI will be performed to obtain data for cerebral blood flow ( $\mathrm{CBF}$ ) in the brain. Voxel-based $\mathrm{CBF}$ will be mapped using local software.

A three-dimensional, T1-weighted image will be acquired using the magnetization-prepared rapid gradient-echo (MPRAGE) sequence to quantify the gray and white matter tissue volume in the brain. T2-weighted turbo-spin echo (TSE) and fluid attenuation inversion recovery (FLAIR) sequences will be used to evaluate brain abnormalities in subjects.

3) Blood test

Amyloid $\beta(A \beta)$ protein, tau protein, high-mobility group box (HMGB) protein, small EDRK-rich factor (SERF) 1A, and cholesterol derivatives will be measured in the blood at baseline and after 24 weeks of KGT or placebo treatment to observe changes in levels of serum proteins and cholesterol associated with $\mathrm{AD}$ pathology. 
The Effect of Adding Kami-guibi-tang to Acetylcholinesterase Inhibitor Treatment on the Cognitive Function of Mild Alzheimer's Disease Patients: Study Protocol of a Randomized, Placebo-Controlled, Double-Blind Pilot Trial

\section{4) Gene test}

The presence of apolipoprotein E (APOE) epsilon (e23, e33, e34, e44) will be identified using blood tests at baseline to observe differences in the effect of KGT between carriers and non-carriers.

5) Safety assessment

Safety assessments will be conducted using laboratory tests and electrocardiography (ECG). A blood test, measuring levels of AST/ALT, glucose, BUN (blood urea nitrogen), creatinine, $\mathrm{Na}, \mathrm{K}, \mathrm{Cl}$, cholesterol, $\mathrm{LDH}$, and CPK, will be performed at baseline and after 12 and 24 weeks of treatment. ECG will be performed at baseline and after 24 weeks of KGT or placebo treatment. Vital signs will be recorded at every visit. A phone call will be made four weeks after completion of treatment to monitor for the any occurrence of adverse events.

\section{Outcome measures}

1) Primary outcome measures

(1) Changes in SNSB scores before and after treatment.

(2) Changes in MRI measurements (brain metabolites, neurotransmitters, cerebral blood flow) before and after treatment.

2) Secondary outcome measures

(1) Safety assessment based on changes in blood chemistry (AST/ALT, glucose, BUN, creatinine, $\mathrm{Na}, \mathrm{K}, \mathrm{Cl}, \mathrm{LDH}, \mathrm{CPK}$ ) and ECG.

(2) Changes in blood proteins and cholesterol (amyloid $\beta$ protein, tau protein, HMGB, SERF $1 \mathrm{~A}$, and cholesterol derivatives) before and after treatment.

(3) Comparisons of changes in MRI measurements (brain metabolites, neurotransmitters, cerebral blood flow) between treatment and placebo groups using age and genotype $(\mathrm{ApoE})$ as covariates.
(4) Changes in KQoL-AD and CGA-NPI scores before and after treatment.

\section{Data management and monitoring}

CRFs will be used for each subject to collect relevant data. To promote data quality and accuracy, one trained investigator will complete the CRFs, and a second investigator will independently review all CRFs. All documents will be kept at the study site, and all data will be entered and stored in a password protected computer. All procedures will comply with confidentiality standards for medical data. All documents and collected data will be kept for three years after completion of the study, and will then be destroyed. The data management process will be monitored by a clinical monitor, who is independent of the research team. Only the investigators and monitor will have access to the dataset. Auditing will be conducted by the Korean Ministry of Food and Drug Safety.

\section{Statistics}

1) Sample size calculation

This clinical trial is a pilot study to examine the feasibility of a full randomized clinical trial of $\mathrm{KGT}$, and to determine the sample magnitude required for large-scale studies. To our knowledge, no prior study has investigated the effect of KGT on mild AD. There are therefore no previous data indicating the sample size needed to yield statistically significant results for determining the effect of KGT as determined by SNSB scores. Normally, 10 to 20 subjects in each group should be sufficient to implement a pilot study. In this study, we plan to perform a statistical analysis using a total of 30 subjects, with 15 in each group. Allowing for a maximum dropout rate of $20 \%$, the desired sample 
size for this pilot study is 38 subjects, with 19 in each group.

2) Data analysis

We will conduct data analysis with the help of an independent, professional statistician. All statistical analyses will be performed using SPSS for Windows. The data will be analyzed according to the intentionto-treat (ITT) principle and per protocol principle. We will adjust for missing data using the last observation carried forward (LOCF) principle to obtain a complete database. We will not perform an interim analysis.

Student's t-test will be used for parametric variable comparisons between groups, and the paired t-test will be used for intra-group comparisons. The Mann-Whitney U test will be used for non-parametric variable comparisons between groups, and the Wilcoxon signed-rank test will be used for intra-group comparisons.

The paired t-test will be used to compare changes in MRI measurements, blood protein levels, and cholesterol levels before and after treatment. A regression analysis will be used to estimate the relationship between changes in MRI measurements and changes in blood tests. The two-sample t-test will be applied to compare changes in MRI measurements between the study group and the placebo group, using age and genotype as covariates.

\section{Adverse events}

Any undesirable, unexpected sign, symptom, or disease that occurs during the trial will be identified as an adverse event ( $\mathrm{AE})$, regardless of its relationship with the study intervention. The occurrence of AEs will be assessed at every visit using subjective self-reports from patients and objective examinations, including blood tests and
ECG. AEs will also be monitored by telephone each week during the intervention period and four weeks after the end of treatment. All AEs will be recorded in the CRF by the site investigator and assessed for severity and causality, and will be reported to patients and other investigators. Details of each type of $\mathrm{AE}$ will be recorded, including starting and ending date, feature, duration, severity, and relationship to the study medication.

Serious adverse events (SAEs) are defined as illnesses requiring hospitalization, events that result in persistent or significant disability or incapacity, events deemed life-threatening, death, a congenital anomaly or birth defect, or other important medical conditions. If SAEs occur, study participation will be discontinued for that subject, appropriate measures will be taken immediately, and the institutional review board (IRB) will be notified as soon as possible.

All AEs that occur will be tracked until they subside or stabilize.

\section{Ethics}

The trial will be carried out in accordance with the Declaration of Helsinki and the Korean Good Clinical Practice Guidelines. It has already been approved by the Institutional Review Board of Kyung Hee University Hospital at Gangdong (KHNMC-OH 2017-11-002-003) and by the Korean Ministry of Food and Drug Safety (31234). The protocol has been registered in Clinical Research Information Service (KCT0002904). Voluntary written informed consent will be obtained from all study participants prior to enrollment in the study. 
The Effect of Adding Kami-guibi-tang to Acetylcholinesterase Inhibitor Treatment on the Cognitive Function of Mild Alzheimer's Disease Patients: Study Protocol of a Randomized, Placebo-Controlled, Double-Blind Pilot Trial

\section{Discussion}

In this study, extensive assessment tools will be used to evaluate the effects of KGT and the mechanisms of any detected effects. Instruments such as the K-MMSE, SNSB, KQoL-AD, and CGA-NPI will be used to assess cognitive function. The MMSE is a short, global test, and is the most widely used instrument for measuring global cognitive function ${ }^{24}$. However, Kang et al. suggested that the K-MMSE is relatively insensitive for detecting the early stages of dementia, resulting in a high level of false negatives ${ }^{25}$. Therefore, we will also use the SNSB-II, which is a valid and reliable tool for assessing overall cognitive function ${ }^{26}$, to assess cognitive function in this trial. The KQoL-AD, which has very good psychometric properties and can be completed in subjects with a wide range of dementia severities ${ }^{27,28}$, will be used to assess quality of life. The CGA-NPI will be used to assess neuropsychiatric symptoms in dementia patients ${ }^{29}$.

MRI is the imaging modality of choice in dementia. Structural MRI-based measurements of brain atrophy are widely accepted markers of $\mathrm{AD}$ progression, but no structural imaging features have perfect sensitivity and specificity for any given diagnosis ${ }^{30}$. Usually, overt loss of neurons and the associated brain atrophy occur in the later stages of $\mathrm{AD}^{31}$, but are occasionally observed in the preclinical stages of cognitive impairment ${ }^{32}$. There is a limit to our ability to explore dynamic neuropathological processes by structural MRI; therefore, metabolic and functional changes in the brain indicated by measures of brain metabolites, neurotransmitters, and cerebral blood flow will be explored in this trial.

Proton MRS will be used to measure brain metabolite and neurotransmitter levels, including NAA, Glx, and GABA. Reduction of NAA is the most frequent $1 \mathrm{H}-\mathrm{MRS}$ finding in $\mathrm{AD}$. Some studies have reported reduced Glx levels in AD patients compared to controls in the posterior cingulate cortex, occipital lobe, and the dominanthemisphere lateral temporal cortex ${ }^{33}$. Cognitive function is sensitive to cerebral GABA concentrations in the frontal cortex ${ }^{34}$.

ASL MRI provides a quantitative measure of cerebral blood flow (CBF). Some studies indicate that $\mathrm{CBF}$ may even be sensitive for predicting cognitive decline and conversion to mild cognitive impairment and/or $\mathrm{AD}$ over time ${ }^{35.36}$. A previous study revealed that treatment with rivastigmine over 24 months prevented a decrease of regional $\mathrm{CBF}$ ( $\mathrm{rCBF}$ ) in patents with $\mathrm{AD}^{37}$.

$A \beta$ protein, tau protein, HMGB protein, SERF $1 \mathrm{~A}$, and cholesterol derivatives will be measured to observe changes in serum protein and cholesterol levels associated with AD pathology. Numerous studies have suggested that $A B$ may mediate adverse effects in the brain by directly altering neuronal function or by forming plaques that induce inflammatory responses, subsequently decreasing neuronal activity and viability ${ }^{38}$. Understanding the regulation of tau phosphorylation is a subject of great interest because it may be involved in the formation of tau aggregates and because it may aid in the elaboration of protective strategies to cope with these lesions in $\mathrm{AD}^{39}$. An in vivo study suggested that the HMGB-1 protein promotes the neuronal differentiation of adult hippocampal neural progenitors via receptors for the advanced glycation end products/nuclear factor- $\kappa \mathrm{B}$ axis that contributes to neuroinflammation/neurotoxicity in $\mathrm{AD}^{40}$.

APOE epsilon will be measured to observe 
differences in the effects of KGT between carriers and non-carriers. The APOE gene is the main genetic risk factor associated with $\mathrm{AD}^{41}$. $\mathrm{APOE} \varepsilon 4$ carriers have enhanced AD pathology, accelerated age-dependent cognitive decline, and worse memory performance than non-carriers ${ }^{42}$.

This pilot study will constitute a rigorous clinical analysis of KGT for the treatment of mild $A D$, and will provide evidence for the effect of adding KGT to AchEI treatment. Our extensive assessment of various biomarkers will provide information regarding the mechanisms underlying any effects of KGT on disease progression. These findings may support large-scale clinical trials to consolidate evidence for KGT use in $\mathrm{AD}$ patients.

\section{Conflict of interest}

Authors declare no conflict of interests.

\section{Funding}

This study is supported by the Convergence of Conventional Medicine and Traditional Korean Medicine R \& D program funded by the Ministry of Health \& Welfare through the Korea Health Industry Development Institute (KHIDI) (grant number: HI16C2352). The funder had no role in the study design, collection, analysis and interpretation of data, writing of the report and in the decision to submit the article for publication.

\section{References}

1. Burns A, Iliffe S. Alzheimer's disease. Bmj 2009; 338:b158.
2. Reisberg B, Franssen EH, Bobinski M, Auer S, Monteiro I, Boksay I, et al. Overview of methodologic issues for pharmacologic trials in mild, moderate, and severe Alzheimer's disease. Int Psychogeriatr 1996;8(2):159-93.

3. Karttunen K, Karppi P, Hiltunen A, Vanhanen M, Välimäki T, Martikainen J, et al. Neuropsychiatric symptoms and quality of life in patients with very mild and mild Alzheimer's disease. Int $J$ Geriatr Psychiatry 2011;26(5):473-82.

4. Wimo A, Jönsson L, Bond J, Prince M, Winblad B. The worldwide economic impact of dementia 2010. Alzheimers Dement 2013:9(1):1-11.e3.

5. O’Brien JT, Holmes C, Jones M, Jones R, Livingston G, McKeith I, et al. Clinical practice with anti-dementia drugs: A revised (third) consensus statement from the British Association for Psychopharmacology. $J$ Psychopharmacol 2017;31(2) :147-68.

6. Eun JS, Song JM. Effects of Kwibi-tang on Serum Levels of Hormone and the Non-Specific Immune Response after Immobilization Stress in Mice. Korean Journal of Oriental Physiology \& Pathology 2004;18(1):172-8.

7. Jeon HJ, Park SW, Lee I, Mun BS. Effects of Gwibitang on Glutamate-induced Death in Rat Neonatal Astrocytes. $J$ Korean Oriental Med 2004;25(2) :184-93.

8. Park IK, Kim KJ. A Study on the Homostatic Effects of Guipitang and Guipitangjiaweifang Extracts in Experimental Animals. The journal of Korean oriental medical ophthalmology \& otolaryngology \& dermatology 2003;16(1):63-76.

9. Cho SI, Jo SH, Jin CS. Effects of the Guipi-Tang and Placenta Hominis Blended Guipi-Tang on the Changes of Body Weight and Lipid Metabolism in Ovariectomized Rats. The Korea journal of 
The Effect of Adding Kami-guibi-tang to Acetylcholinesterase Inhibitor Treatment on the Cognitive Function of Mild Alzheimer's Disease Patients: Study Protocol of a Randomized, Placebo-Controlled, Double-Blind Pilot Trial

herbology 2004;19(3):85-90.

10. Kim HJ, Choi JH, Lim SW. The Defensive Effect of Keuibi-tang on the Gastric Mucous Membrane of Mouse Injured by Stress and Ethanol. Journal of Korean Medicine 2003;24(1) :155-68.

11. Jung YC, Kim DC, Back SH, Kim EH. The Effect of Guibitang(歸脾湯) on the Ovarian Functions and Differential Gene Expression of Caspase-3, MAPK and MPG in Female Mice. The Journal of Korean Obstetrics \& Gynecology 2007;20(3) :13-34.

12. Oh MS, Huh Y, Bae H, Ahn DK, Park SK. The multi-herbal formula Guibi-tang enhances memory and increases cell proliferation in the rat hippocampus. Neuroscience letters 2005:379(3) :205-8.

13. Kim SH, Lee SE, Oh H, Yang JA, Chung CY, Jang JS, et al. The Radioprotective Effect of Kuei-Pi-Tang as a prescription of Traditional Chinese Medicine in Mice. Journal of the Korean Society of Food Science and Nutrition 1999; 28(3) :698-704.

14. Kim JH, Lee JK, Ha HK, Seo CS, Lee MY, Lee HY, et al. Analysis of Studies on Guibi-tang (Guipitang) for Fundamental Establishment of Evidence Based Medicine(EBM). Journal of Oriental Neuropsychiatry 2009;20(3):205-16.

15. Ishida K. Effect of donepezil and kamikihito combination therapy on cognitive function in A lzheimer's disease: $R$ etrospective study. Traditional \& Kampo Medicine 2016:3(2):94-9.

16. 馬込敦. 認知症に對する加味歸脾湯の効果. 漢方 と最新治療 2014;23(2) :135-40.

17. Egashira N, Manome N, Kurauchi K, Matsumoto Y, Iwasaki K, Mishima K, et al. Kamikihi-to, a Kampo medicine, ameliorates impairment of spatial memory in rats. Phytother Res 2007; 21(2) :126-9.

18. Tohda C, Nakada R, Urano T, Okonogi A, Kuboyama T. Kamikihi-to (KKT) rescues axonal and synaptic degeneration associated with memory impairment in a mouse model of Alzheimer's disease, 5XFAD. Int $J$ Neurosci 2011;121(12):641-8.

19. Lee JW, Cho DG, Cho WS, Ahn HG, Lee HJ, Shin JW, et al. Effect of Guibi-tang on Neuronal Apoptosis and Cognitive Impairment Induced by Beta Amyloid in Mice. Journal of Korean medicine 2014;35(4):10-23.

20. Qian YH, Han H, Hu XD, Shi LL. Protective effect of ginsenoside $\mathrm{Rb} 1$ on beta-amyloid protein(1-42)-induced neurotoxicity in cortical neurons. Neurol Res 2009:31(7):663-7.

21. Zhang H, Han T, Zhang L, Yu CH, Wan DG, Rahman K, et al. Effects of tenuifolin extracted from radix polygalae on learning and memory: a behavioral and biochemical study on aged and amnesic mice. Phytomedicine 2008;15(8):587-94.

22. Nogami-Hara A, Nagao M, Takasaki K, Egashira N, Fujikawa R, Kubota K, et al. The Japanese Angelica acutiloba root and yokukansan increase hippocampal acetylcholine level, prevent apoptosis and improve memory in a rat model of repeated cerebral ischemia. J Ethnopharmacol 2018;214 :190-6.

23. Cho MJ, Kim JH, Park CH, Lee AY, Shin YS, Lee JH, et al. Comparison of the effect of three licorice varieties on cognitive improvement via an amelioration of neuroinflammation in lipopolysaccharide-induced mice. Nutr Res Pract 2018;12(3) :191-8.

24. Folstein MF, Folstein SE, McHugh PR. "Mini-mental state". A practical method for grading the 
cognitive state of patients for the clinician. $J$ Psychiatr Res 1975;12(3):189-98.

25. A validity study on the korean mini-mental state examination (K-MMSE) in dementia patients. $J$ Korean Neurol Assoc 1997;15(2):300-8.

26. Kang Y, Jang SM, Na DL. Seoul Neuropsychological Screening Battery (SNSB-II) 2nd ed. Seoul: Human Brain Research \& Consulting Co.; 2012.

27. Thorgrimsen L, Selwood A, Spector A, Royan L, de Madariaga Lopez M, Woods RT, et al. Whose quality of life is it anyway? The validity and reliability of the Quality of Life-Alzheimer's Disease (QoL-AD) scale. Alzheimer Dis Assoc Disord 2003;17(4):201-8.

28. Shin HY. A preliminary study on the Korean version of quality of life-Alzheimer's disease (QOL-AD) scale in community-dwelling elderly with dementia. J Prev Med Public Health 2006; $39(3): 243-8$.

29. Kang SJ, Choi SH, Lee BH, Jeong Y, Hahm DS, Han IW, et al. Caregiver-Administered Neuropsychiatric Inventory (CGA-NPI), $J$ Geriatr Psychiatry Neurol 2004;17(1):32-5.

30. Harper L, Barkhof F, Scheltens P, Schott JM, Fox NC. An algorithmic approach to structural imaging in dementia. $J$ Neurol Neurosurg Psychiatry 2014;85(6):692-8.

31. Citron M. Alzheimer's disease: strategies for disease modification. Nat Rev Drug Discov 2010;9(5) :387-98.

32. Hilal S, Amin SM, Venketasubramanian N Niessen WJ, Vrooman H, Wong TY, et al. Subcortical Atrophy in Cognitive Impairment and Dementia. J Alzheimers Dis 2015;48(3) :813-23.

33. Griffith HR, Stewart CC, den Hollander JA. Proton magnetic resonance spectroscopy in dementias and mild cognitive impairment. Int Rev Neurobiol 2009;84:105-31.

34. Porges EC, Woods AJ, Edden RA, Puts NA, Harris AD, Chen $\mathrm{H}$, et al. Frontal GammaAminobutyric Acid Concentrations Are Associated With Cognitive Performance in Older Adults. Biol Psychiatry Cogn Neurosci Neuroimaging 2017;2(1) :38-44.

35. Beason-Held LL, Goh JO, An Y, Kraut MA, O’Brien RJ, Ferrucci L, et al. Changes in brain function occur years before the onset of cognitive impairment. $J$ Neurosci 2013:33(46) :18008-14

36. Chao LL, Buckley ST, Kornak J, Schuff N, Madison C, Yaffe K, et al. ASL perfusion MRI predicts cognitive decline and conversion from MCI to dementia. Alzheimer Dis Assoc Disord 2010:24(1):19-27.

37. Lipczyńska-Kojkowska W, Ryglewicz D, Jedrzejczak T, Jakubowska T, Kotapka-Minc S, Sienkiewicz-Jarosz $\mathrm{H}$, et al. The effect of rivastigmine on cognitive functions and regional cerebral blood flow in Alzheimer's disease and vascular dementia: follow-up for 2 years. Neurol Neurochir Pol 2004;38(6) :471-81.

38. Walsh DM, Teplow DB. Alzheimer's disease and the amyloid $\beta$-protein. Prog Mol Biol Transl Sci 2012;107:101-24.

39. Martin L, Latypova X, Wilson CM, Magnaudeix A, Perrin ML, Yardin C, et al. Tau protein kinases: involvement in Alzheimer's disease. Ageing Res Rev 2013;12(1):289-309.

40. Meneghini V, Bortolotto V, Francese MT, Dellarole A, Carraro L, Terzieva S, et al. High-mobility group box-1 protein and $\beta$-amyloid oligomers promote neuronal differentiation of adult hippocampal neural progenitors via receptor for advanced 
The Effect of Adding Kami-guibi-tang to Acetylcholinesterase Inhibitor Treatment on the Cognitive Function of Mild Alzheimer's Disease Patients: Study Protocol of a Randomized, Placebo-Controlled, Double-Blind Pilot Trial

glycation end products/nuclear factor- ${ }^{\kappa} \mathrm{B}$ axis: relevance for Alzheimer's disease. $J$ Neurosci 2013:33(14):6047-59.

41. Corder EH, Saunders AM, Strittmatter WJ, Schmechel DE, Gaskell PC, Small GW, et al. Gene dose of apolipoprotein E type 4 allele and the risk of Alzheimer's disease in late onset families. Science 1993;261(5123):921-3.

42. Kok E, Haikonen S, Luoto T, Huhtala H, Goebeler S, Haapasalo H, et al. Apolipoprotein E-dependent accumulation of Alzheimer diseaserelated lesions begins in middle age. Ann Neurol 2009:65(6):650-7. 